\title{
Valence band mixing of cubic GaN/AIN quantum dots
}

\author{
C. Segarra, J.I. Climente and J. Planelles \\ Departament de Química Física i Analítica, Universitat Jaume I, E-12080, Castelló, \\ Spain \\ E-mail: climente@uji.es
}

\begin{abstract}
.
We study the spin purity of the hole ground state in nearly axially symmetric GaN/AlN quantum dots (QDs). To this end, we develop a six-band Burt-Foreman Hamiltonian describing the valence band structure of zinc-blende nanostructures with cylindrical symmetry, and calculate the effects of eccentricity variationally. We show that that the aspect ratio is a key factor for spin purity. In typical QDs with small aspect ratio the ground state is essentially a heavy hole $(\mathrm{HH})$ whose spin purity is even higher than that of InGaAs QDs of similar size. When the aspect ratio increases, mixing with light-hole (LH) and split-off (SO) subbands becomes important and, additionally, the ground state becomes sensitive to QD anisotropy, which further enhances the mixing. We finally show that despite the large GaN hole effective mass, an efficient magnetic modulation is feasible in QDs with aspect ratio $\sim 1$, which can be used to modify the ground state symmetry and hence the optical spectrum properties.
\end{abstract}

PACS numbers: 73.21.La, 75.75.-c, 78.55.Cr

Submitted to: J. Phys.: Condens. Matter 


\section{Introduction}

GaN/AlN QDs are nanostructures of current interest for optoelectronic applications owing to their emission in the UV spectrum and their efficient optical activity up to room temperature.[1] The former property follows from the wide band gap of GaN (3.4 $\mathrm{eV}$ ), while the latter follows from the low dielectric constants, large effective masses and band-offsets, which enable unprecedented strength of exciton confinement. The wide band gap is also responsible for weak spin-orbit interactions, [2] which should translate into long exciton spin relaxation lifetimes. This is of interest for spintronic applications.

GaN QDs can be grown in hexagonal (wurtzite) or cubic (zinc-blende) crystallographic phases.[1, 3, 4] Wurtzite QDs are characterized by the presence of strong built-in electric fields (of the order of $\mathrm{MV} / \mathrm{cm}$ ) due to spontaneous and piezoelectric polarization. [1, 5] This constitutes a critical factor in determining the optical response of the $\mathrm{QDs}[6,5,7]$, as well as the exciton spin lifetime, which turns out to be rather short - of the order of 200 ps at room temperature--[8] Built-in electric fields are however missing in GaN/AlN QDs with zinc-blende structure.[6] Lagarde et al. showed that, as a consequence, the optical orientation in cubic structures is robust even at room temperature, with exciton spin lifetimes exceeding 10 ns.[9]

These results hold promise for both optoelectronic and spintronic applications of cubic GaN/AlN QDs, and have triggered an increasing number of works investigating their properties.[2, 10, 11, 12, 13] An important aspect to understand such properties is the valence band mixing, which is known to underlie the optical polarization[13, 14] and the exciton spin dynamics. $[13,15,16]$ The valence band structure of GaN is complicated because the spin-orbit splitting is only $17 \mathrm{meV}$.[17] As a consequence, light-hole (LH) and split-off (SO) subbands may couple strongly and come close to the heavy-hole (HH) subband in the Brillouin zone center, as noted in GaN/AlN superlattices.[18] The situation could however be different in QDs because HH, LH and SO have different effective masses and hence feel quantum confinement differently.[13] Indeed, the long spin lifetimes observed by Lagarde et al. suggest a ground state with weak valence band mixing. Understanding the relationship between QD confinement and valence band mixing is then desirable.

In this work we investigate how the size and shape of cubic GaN/AlN QDs influences the valence band admixture of the hole ground state. The QDs are assumed to be grown along the [001] axis. [3, 4] Because holes have strongly anisotropic masses, we find that flat QDs -where vertical confinement dominates over lateral one- favor HH character and high spin purity. As a matter of fact, the spin purity is higher than that of more conventional materials as InGaAs, which supports the suitability of these structures for optical spin storage. By contrast, high QDs with strong lateral confinement imply dominant LH character. When vertical and lateral confinements are comparable $\mathrm{HH}$ and LH states are close in energy. Then, the admixture becomes significant and very sensitive to in-plane anisotropy, as noted in recent experiments. In this case, we show that the different Zeeman splitting of states with dominant $\mathrm{HH}$ and LH components can 
be used to induce ground state transitions. This enables efficient magnetic manipulation of the optical spectrum in spite of the large effective mass of GaN.

\section{Theory}

An accurate description of holes in GaN/AlN QDs can be obtained using 6-band $\mathrm{k} \cdot \mathrm{p}$ Hamiltonians including HH, LH and SO subbands.[19] This requires spanning the Hamiltonian on the basis of periodic Bloch functions $\left|J, J_{z}\right\rangle$ :

$$
\begin{aligned}
& \left|\frac{3}{2},+\frac{3}{2}\right\rangle=\frac{1}{\sqrt{2}}|(X+i Y) \uparrow\rangle=\left|h h_{+}\right\rangle, \\
& \left|\frac{3}{2},+\frac{1}{2}\right\rangle=\frac{1}{\sqrt{6}}|(X+i Y) \downarrow\rangle-\sqrt{\frac{2}{3}}|Z \uparrow\rangle=\left|l h_{+}\right\rangle, \\
& \left|\frac{3}{2},-\frac{1}{2}\right\rangle=-\frac{1}{\sqrt{6}}|(X-i Y) \uparrow\rangle-\sqrt{\frac{2}{3}}|Z \downarrow\rangle=\left|l h_{-}\right\rangle, \\
& \left|\frac{3}{2},-\frac{3}{2}\right\rangle=\frac{1}{\sqrt{2}}|(X-i Y) \downarrow\rangle=\left|h h_{-}\right\rangle, \\
& \left|\frac{1}{2},+\frac{1}{2}\right\rangle=\frac{1}{\sqrt{3}}|(X+i Y) \downarrow\rangle+\sqrt{\frac{1}{3}}|Z \uparrow\rangle=\left|s o_{+}\right\rangle, \\
& \left|\frac{1}{2},-\frac{1}{2}\right\rangle=-\frac{1}{\sqrt{3}}|(X-i Y) \uparrow\rangle+\sqrt{\frac{1}{3}}|Z \downarrow\rangle=\left|s o_{-}\right\rangle .
\end{aligned}
$$

The $|3 / 2, \pm 3 / 2\rangle$ components correspond to $\mathrm{HH}$, the $|3 / 2, \pm 1 / 2\rangle$ to $\mathrm{LH}$ and the $|1 / 2, \pm 1 / 2\rangle$ to SO. One can see from the explicit $\left|J, J_{z}\right\rangle$ functions above that $\mathrm{HH}$ components have pure spin, while LH and SO components contain spin admixture.

Since the Luttinger parameters of GaN and AlN are quite different, it is convenient to employ position-dependent effective mass parameters. Then, instead of the classical Luttinger Hamiltonian[20] one must use the Burt-Foreman one.[21, 22] A detailed description of this Hamiltonian can be found in Ref. [23], where the due expression in cartesian coordinates is given.[24] For circular QDs it is however convenient to use cylindrical coordinates instead. We then convert the coordinate system from cartesian to cylindrical. Additionally, we include a magnetic field along [001] by following the prescription of Ref. [25] i.e., by introducing the magnetic terms in the k·p Hamiltonian prior to applying the envelope function approximation. Note that this is contrary to the traditional Luttinger formulation for bulk semiconductors and usual formulations for nanostructures which implement the magnetic field after the envelope function approximation.[26] For multi-band studies of nanostructures, our formulation provides a more reliable description of the magnetic field.[27, 28]

The resulting Hamiltonian is one of the important results of this work. It is a 6-by-6 matrix, $\mathcal{H}_{6}$, whose elements are given in the Appendix. The QD is modeled as a quantum disk of radius $R$ and height $H$. Since the disk has axial symmetry, the angular coordinate is integrated analytically. Then, within the axial approximation of 
the k.p Hamiltonian,[29] the states can be labeled by their total angular momentum $F_{z}=m_{z}+J_{z}$, which is the sum of the envelope angular momentum $m_{z}$ and the Bloch angular momentum $J_{z}$. The eigenfunctions of $\mathcal{H}_{6}$ are then six-component spinorial objects of the form:

$$
\left|F_{z}, n\right\rangle=\left(\begin{array}{cc}
f_{F_{z}-3 / 2}^{(1)}(\rho, z) & \left|h h_{+}\right\rangle \\
f_{F_{z}-1 / 2}^{(2)}(\rho, z) & \left|l h_{+}\right\rangle \\
f_{F_{z}+1 / 2}^{(3)}(\rho, z) & \left|l h_{-}\right\rangle \\
f_{F_{z}+3 / 2}^{(4)}(\rho, z) & \left|h h_{-}\right\rangle \\
f_{F_{z}-1 / 2}^{(5)}(\rho, z) & \left|s o_{+}\right\rangle \\
f_{F_{z}+1 / 2}^{(6)}(\rho, z) & \left|s o_{-}\right\rangle
\end{array}\right)
$$

where $n=1,2,3 \ldots$ is the main quantum number and $f_{m_{z}}^{(i)}(\rho, z)$ is the envelope function of the $i$-th component. For calculations in this work we use GaN and AlN material parameters.[17] The confining potential is zero inside the QD and $V_{0}$ outside, where $V_{0}=0.5 \mathrm{eV}$ is the valence band-offset between GaN and AlN.[30] For InGaAs/GaAs QDs, which we also study for comparison, we take $\operatorname{In}_{0.53} \mathrm{Ga}_{0.47}$ As and GaAs parameters, with $V_{0}=0.4 \mathrm{eV}$.[17] For simplicity, strain is disregarded. This leads to slightly overestimated subband mixing, but the trends we report should not be affected. The Hamiltonian is integrated with a finite differences scheme.

\section{Results and discussion}

In this section we investigate the composition of the hole ground state as a function of the QD geometry and external fields. The composition is given in terms of the weight of each component within the spinor (1). For example, the weight of the $\left|h h_{+}\right\rangle$component is:

$$
c_{h h_{+}}=\frac{\left\langle f^{(1)} \mid f^{(1)}\right\rangle}{\sum_{i}\left\langle f^{(i)} \mid f^{(i)}\right\rangle} .
$$

\subsection{Effect of the aspect ratio}

Our starting point is a GaN QD with typical dimensions, radius $R=6 \mathrm{~nm}$ and height $H=1.5 \mathrm{~nm}$.[3, 9] The ground state has $F_{z}=3 / 2$ symmetry, with a largely dominant $\left|h h_{+}\right\rangle$component.[31] Yet, the minor components are important in determining the optical polarization and the hole spin dynamics.[13] Thus, in Fig. 1(a) we analyze how the minor components vary with the QD radius (solid lines). For comparison, we also show the minor components in the better-known case of InGaAs/GaAs QDs (dashed lines), which is taken as a reference. One can see that in both GaN and InGaAs QDs the weight of the minor components decreases with $R$.

This result can be understood from the anisotropic effective masses of holes, which are summarized in Table 1. In the QDs of Fig. 1(a), the vertical confinement is 

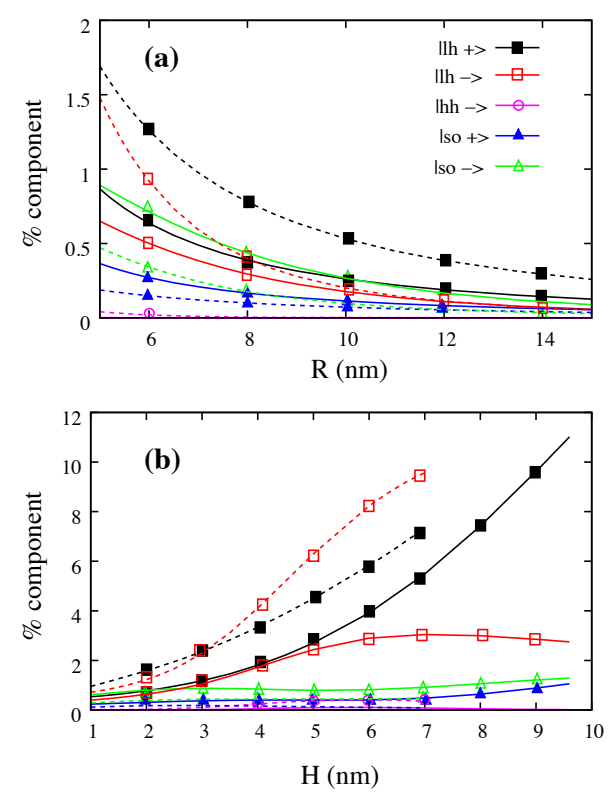

Figure 1. (Color online). Minor components of the hole ground state in GaN/AlN QDs (solid lines) and InGaAs/GaAs QDs (dashed lines). (a) Variable radius and fixed height $H=1.5 \mathrm{~nm}$. (b) Variable height and fixed radius $R=6 \mathrm{~nm}$.

much stronger than the lateral one. If we disregard lateral confinement completely and pay attention to the effective masses along $z$ ([001] axis) only, we can see that $m_{h h}^{z}>m_{l h}^{z} \sim m_{s o}$. Thus, the kinetic energy of LH and SO states will be large and coupling with $\mathrm{HH}$ weak. The smaller the aspect ratio $(\mathrm{H} / 2 \mathrm{R})$, the closer we are to this limit.

In Fig. 1(b) we plot the variation of the minor components with the QD height. Here the behavior is the opposite. As $H$ increases the vertical confinement becomes weaker. Then, the lateral confinement becomes more relevant and the ground state gains LH character because $m_{l h}^{\perp}>m_{h h}^{\perp}$. As a result, the $\mid l h_{+}>$component weight may now exceed $10 \%$ for large $H$. As a matter of fact, when $H$ is large enough the ground state symmetry changes from $F_{z}= \pm 3 / 2$ (dominant $\mathrm{HH}$ component) to $F_{z}= \pm 1 / 2$ (dominant LH component). This translates into a sharp enhancement of the LH character, which can be used to emit strongly linearly polarized light.[32, 33, 34] For InGaAs QDs the transition occurs at $H=7 \mathrm{~nm}$ (aspect ratio $\sim 0.6$ ), while for GaN QDs it occurs at $H=9.7 \mathrm{~nm}$ (aspect ratio $\sim 0.8$ ). For clarity of presentation, in Fig. 1 we have truncated the lines at the position of the transitions. State-of-the-art cubic GaN QDs are grown by self-assembly techniques and have small aspect ratio. Yet, the results in Fig. 1(b) stress the interest of potential developments in the synthesis of elongated QDs.

Fig. 1 reveals that the valence band mixing of the ground state in GaN QDs is weaker than that in InGaAs QDs with equal size. This implies high spin purity, which is consistent with the long spin lifetimes observed by Lagarde et al.[9] The result is however surprising because the effective masses in GaN are much heavier than in InGaAs, so that 
Table 1. Effective masses of HH, LH and SO (times $m_{0}$ ).

\begin{tabular}{cccccc} 
& $m_{h h}^{z}$ & $m_{l h}^{z}$ & $m_{h h}^{\perp}$ & $m_{l h}^{\perp}$ & $m_{s o}$ \\
\hline GaN & 0.85 & 0.24 & 0.29 & 0.52 & 0.37 \\
\hline InGaAs & 0.38 & 0.05 & 0.07 & 0.15 & 0.09
\end{tabular}

the density of states is larger and one could expect stronger mixing. Also, the LH-SO coupling could in principle bring these subbands close to the $\mathrm{HH}$ one, as in higherdimensional structures.[18] The underlying reason for the high purity of the ground state is two-fold. First, the inter-subband coupling terms are weaker than those of InGaAs. For example, many coupling terms are proportional to $\tilde{\gamma}$ (see $\mathcal{H}_{6}$ terms in Appendix). For GaN $\tilde{\gamma}=0.925$, which is about five times smaller than that of InGaAs, $\tilde{\gamma}=4.51$. Second, according to Eq. 1 , the spinor of the $F_{z}=3 / 2$ ground state reads:

$$
|3 / 2,1\rangle=\left(\begin{array}{cc}
f_{0}^{(1)}(\rho, z) & \left|h h_{+}\right\rangle \\
f_{1}^{(2)}(\rho, z) & \left|l h_{+}\right\rangle \\
f_{2}^{(3)}(\rho, z) & \left|l h_{-}\right\rangle \\
f_{3}^{(4)}(\rho, z) & \left|h h_{-}\right\rangle \\
f_{1}^{(5)}(\rho, z) & \left|s o_{+}\right\rangle \\
f_{2}^{(6)}(\rho, z) & \left|s o_{-}\right\rangle
\end{array}\right) .
$$

Note that only the dominant $\left|h h_{+}\right\rangle$component has envelope angular momentum $m_{z}=0$. Other components have finite $m_{z}$ and are then pushed high in energy by the lateral confinement. We stress that this makes valence band mixing in GaN QDs much weaker than in quantum wells.[18]

\subsection{Magnetic field modulation}

The large effective mass of GaN hinders the use of magnetic fields to manipulate the electronic structure of typical QDs (aspect ratio $\sim 1 / 8$ ). To circumvent this problem, consider a GaN QD with aspect ratio close to 1 . In this case the kinetic energy of $\mathrm{HH}$ and $\mathrm{LH}$ is similar. As a consequence, spinors with dominant $\mathrm{HH}$ and $\mathrm{LH}$ character are close in energy and moderate Zeeman splittings suffice to modify the electronic structure. This opens the possibility of magnetic modulation in GaN QDs.

To illustrate this point, in Fig. 2 we show the energy structure of a QD with $R=6$ $\mathrm{nm}$ and $H=10 \mathrm{~nm}$. At zero magnetic field, the ground state is $|1 / 2,1\rangle$ and the first excited one is $|3 / 2,1\rangle$. The dominant components of these spinors are $\left|l h_{+}\right\rangle$and $\left|h h_{+}\right\rangle$, respectively (i.e., the components with $m_{z}=0$ ). The corresponding linear-in- $B$ coefficients are $\left(\gamma_{1}+\gamma_{2}\right) / 2$ for $\left|h h_{+}\right\rangle$and $\left(\gamma_{1}-\gamma_{2}\right) / 6$ for $\left|l h_{+}\right\rangle$-see $\mathcal{H}_{6}$ in Appendix-. Thus, the orbital Zeeman splitting of $|3 / 2,1\rangle$ is larger than that of $|1 / 2,1\rangle$. As a result, with increasing $B$ the ground state changes from $F_{z}=1 / 2$ to $F_{z}=3 / 2$ (see arrow in Fig 2). Because $|3 / 2\rangle$ and $|1 / 2\rangle$ yield different optical polarizations, this can be used to modify the optical response of QDs at will. 


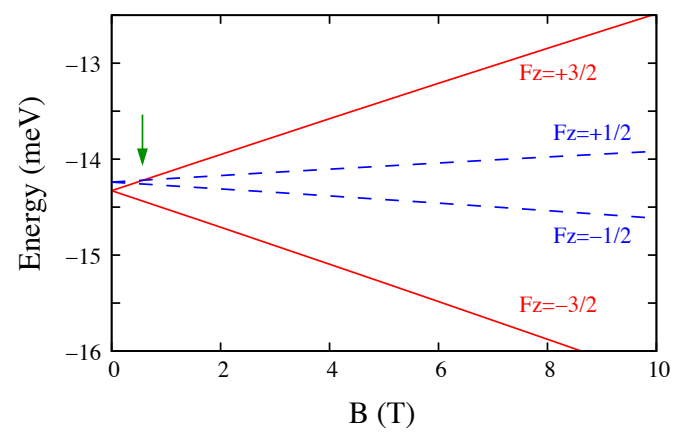

Figure 2. (Color online). Magnetic field splitting of the lowest hole levels in a GaN/AlN QD with aspect ratio $\sim 1$. The arrow points at the ground state transition at $B=0.6 \mathrm{~T}$. Zero energy is the top of the valence band.

\subsection{Effect of $Q D$ anisotropy}

The presence of anisotropy in QDs is often considered to be a source of HH-LH coupling, with due consequences on the optical polarization[36, 37, 38] and hole spin mixing.[39] To see how this affects GaN QDs, next we study how the ground state composition is influenced by an elongation of the QD.

We consider three reference geometries: a QD with typical dimensions, $R=6 \mathrm{~nm}$ and $H=1.5 \mathrm{~nm}$ (QD1); a QD with large aspect ratio - similar to that of InAs QDs-$R=15 \mathrm{~nm}$ and $H=1.5 \mathrm{~nm}$ (QD2); a QD with aspect ratio $\sim 1, R=6 \mathrm{~nm}$ and $H=10$ $\mathrm{nm}$ (QD3). We start from circular QDs and let the eccentricity $\varepsilon$ increase while keeping the basis area constant. The semi-major (semi-minor) axis $R_{a}\left(R_{b}\right)$ of the elliptical QD is then:

$$
\begin{aligned}
& R_{a}=R /\left(1-\varepsilon^{2}\right)^{1 / 4}, \\
& R_{b}=R^{2} / R_{a} .
\end{aligned}
$$

Note from the above expressions that for small QD radius $R$, large eccentricities are required to provide significant anisotropy $R_{a} / R_{b}$. The hole states are calculated with a variational procedure, projecting the $3 \mathrm{D}$ anisotropic potential on a basis of circular $\mathrm{QD}$ eigenstates, as described in Ref. [39] For simplicity, in this section GaN effective mass in used all over the structure.

Figure 3 shows the composition of the ground state in each QD. In QD1 the dominant component is by far $\left|h h_{+}\right\rangle$, with the eccentricity having little effect up to $\varepsilon \sim 0.6$. At this point the semi-minor axis starts imposing a strong lateral confinement and the valence band mixing rapidly increases. For $\varepsilon \sim 0.8\left(R_{b}=4.6 \mathrm{~nm}\right)$ the weight of $\left|h h_{+}\right\rangle$has already decreased from $97 \%$ to $86 \%$. Noteworthily, the largest of the minor components is not a LH but a SO instead - LHs are unfavored by the strong vertical confinement-. In QD2 the dot radius is much larger. As a result, lateral confinement is weak even for strong eccentricities and the ground state composition is barely affected 
by the anisotropy. In QD3 the vertical confinement is weak, so the ground state is $\left|F_{z}=1 / 2, n=1\right\rangle$ with dominant $\left|l h_{+}\right\rangle$component. In this case, even small anisotropies induce sever HH-LH mixing.
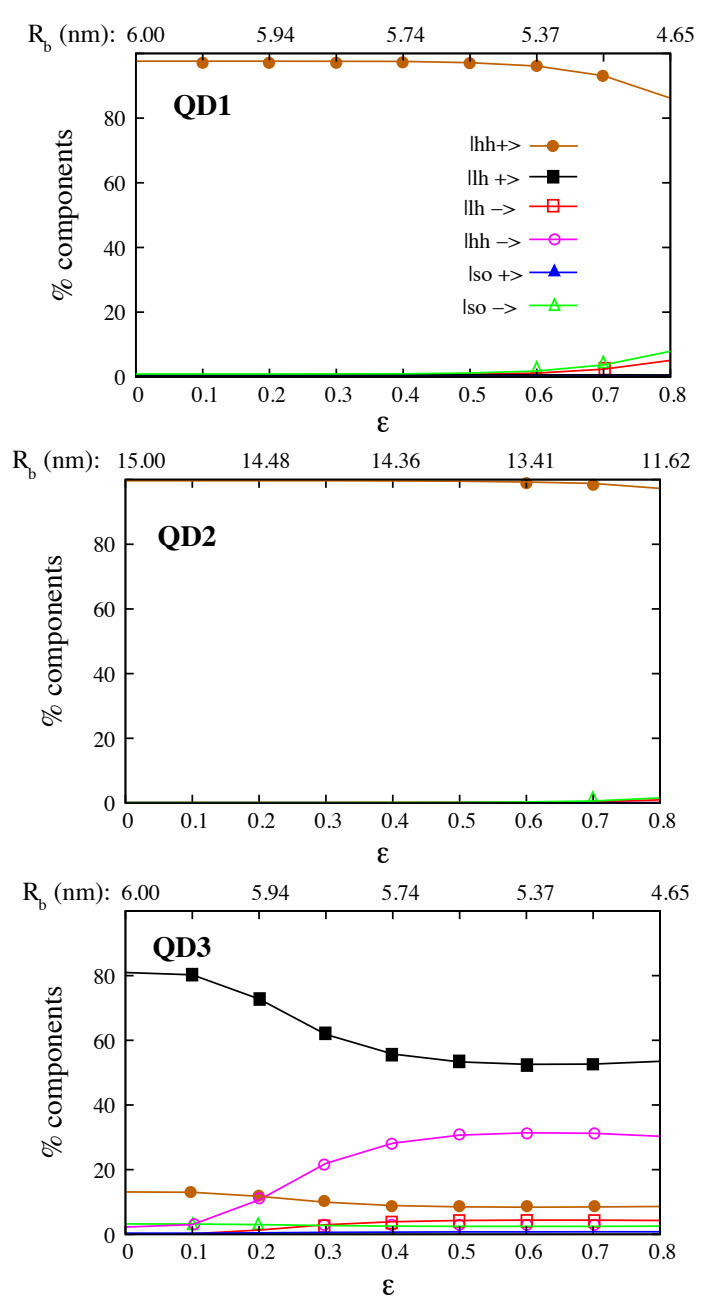

Figure 3. (Color online). Components of the hole ground state as a function of the eccentricity in a typical QD (QD1), a QD with small (QD2) and large (QD3) aspect ratio. The upper axis indicates the length of the semi-minor axis.

Comparing QD1, QD2 and QD3 we conclude that the influence of elongations on the valence band mixing depends on the aspect ratio. When the aspect ratio is small (QD2) the influence is negligible, when it is large (QD3) the influence becomes dramatic. This result is consistent with recent experiments in GaAs QDs, where severe HH-LH mixing was ascribed to dot elongations.[38] Such QDs turn out to have comparable lateral and vertical dimensions.[40] Typical cubic GaN/AlN QDs (QD1) have aspect ratio $\sim 1 / 8$. Owing to the dense energy spectrum this is enough to be sensitive to moderate anisotropies. 


\section{Conclusions}

We have derived a 6-band Burt-Foreman Hamiltonian in cylindrical coordinates for zincblende nanostructures grown along the [001] axis. The Hamiltonian properly includes position-dependent Luttinger parameters and axial magnetic fields.

Using this Hamiltonian we have shown that HH mixing with LH and SO subbands in typical GaN/AlN QDs is weak provided the dot has good circular symmetry. Indeed, the mixing is weaker than that in GaAs/AlAs quantum wells or InAs/GaAs QDs of similar size. This makes the system suited for optical manipulation and storage of spins. Elongations of the QD do however introduce significant HH-SO and HH-LH mixing. The band mixing and the sensitivity to QD anisotropy can be enhanced (reduced) by growing QDs with small (large) aspect ratio.

We have also shown that in GaN QDs with large aspect ratio the small energy splitting between states with dominant $\mathrm{HH}$ and LH components, along with their different Zeeman splittings, can be used to switch the ground state symmetry with external magnetic fields. This is in spite of the large effective masses of GaN, and allows to modify the optical emission characteristics (energy, polarization, intensity).

\section{Acknowledgments}

We thank T. Amand for useful discussions. Support from MICINN projects CTQ200803344 and CTQ2011-27324, UJI-Bancaixa project P1-1A2009-03 and the Ramon y Cajal program (JIC) is acknowledged.

\section{Appendix A. 6-band k·p Hamiltonian}

In this appendix we provide the elements of the Burt-Foreman 6-band Hamiltonian in cylindrical coordinates. An external magnetic field $B$ along the growth axis is included following Ref. [25].

To describe the uniform axial magnetic field, a potencial vector in the symmetric gauge $A=[-y, x, 0] B / 2$ is considered. The presence of this potential vector turns the in-plane part of the kinetic energy operator from $p_{\perp} \frac{1}{2 m_{\perp}} p_{\perp}$ into $\left(p_{\perp}-q A_{\perp}\right) \frac{1}{2 m_{\perp}}\left(p_{\perp}-q A_{\perp}\right)$, where the charge $q=1$ a.u. for holes. In the presence of axial symmetry $m_{\perp}=m_{\perp}(\rho, z)$ and we have,

$$
\mathcal{H}(B)=\mathcal{H}_{0}-\frac{A_{\perp}}{m_{\perp}} p_{\perp}+\frac{A_{\perp}^{2}}{2 m_{\perp}}=\mathcal{H}_{0}+\frac{B}{2 m_{\perp}} L_{z}+\frac{B^{2} \rho^{2}}{8 m_{\perp}}
$$

where $\mathcal{H}_{0}$ is the Hamiltonian in the absence of magnetic field. Now, we follow the procedure in Refs. $[25,28]$ to obtain the magnetic field contribution to the different matrix elements of the Burt-Foreman Hamiltonian. For exemple, the magnetic contribution to the $(1,1)$ matrix element is $\frac{1}{m_{\perp}}\left[\frac{F_{z}-1 / 2}{2} B+\frac{B^{2} \rho^{2}}{8}\right]$, with $m_{\perp}^{-1}=-\left(\gamma_{1}+\gamma_{2}\right)$ being the mass factor corresponding to the $\left|\frac{3}{2}, \frac{3}{2}\right\rangle$ heavy hole state. 
As a result, the position-dependent 6-band Hamiltonian, including and axial uniform magnetic field, reads in cylindrical coordinates as follows:

$$
\mathcal{H}_{6}=\frac{1}{2} \mathcal{M}+V(\rho, z) \mathcal{I}
$$

where atomic units are used $\left(\hbar=q=m_{0}=1\right)$, with $m_{0}$ as the free electron mass. $V(\rho, z)$ is the confining potential, $\mathcal{I}$ is the identity matrix and $\mathcal{M}$ a rank-6 matrix with the following elements:

$$
\begin{aligned}
& \mathcal{M}[1,1]=\frac{\partial}{\partial \rho}\left(\gamma_{1}+\gamma_{2}\right) \frac{\partial}{\partial \rho}+\frac{\left(\gamma_{1}+\gamma_{2}\right)}{\rho} \frac{\partial}{\partial \rho}+\frac{\partial}{\partial z}\left(\gamma_{1}-2 \gamma_{2}\right) \frac{\partial}{\partial z}-\frac{\left(F_{z}-\frac{3}{2}\right)^{2}}{\rho^{2}}\left(\gamma_{1}+\gamma_{2}\right)+ \\
& \frac{\left(F_{z}-\frac{3}{2}\right)}{2 \rho}\left[\frac{\partial}{\partial \rho}\left(C_{1}+C_{2}\right)-\left(C_{1}+C_{2}\right) \frac{\partial}{\partial \rho}\right]-2\left(\gamma_{1}+\gamma_{2}\right)\left[\frac{\left(F_{z}-\frac{1}{2}\right) B}{2}+\frac{B^{2} \rho^{2}}{8}\right], \\
& \mathcal{M}[1,2]=\frac{1}{\sqrt{3}}\left\{\frac{\partial}{\partial \rho} C_{1} \frac{\partial}{\partial z}-\frac{\partial}{\partial z} C_{2} \frac{\partial}{\partial \rho}+\frac{\left(F_{z}-\frac{1}{2}\right)}{\rho}\left[C_{1} \frac{\partial}{\partial z}-\frac{\partial}{\partial z} C_{2}\right]\right\}, \\
& \mathcal{M}[1,3]=-\sqrt{3}\left\{\frac{\partial}{\partial \rho} \tilde{\gamma} \frac{\partial}{\partial \rho}+\frac{\left(F_{z}+\frac{1}{2}\right)}{\rho} \frac{\partial}{\partial \rho} \tilde{\gamma}+\frac{\left(F_{z}-\frac{1}{2}\right)}{\rho} \tilde{\gamma} \frac{\partial}{\partial \rho}+\frac{\left(F_{z}-\frac{3}{2}\right)\left(F_{z}+\frac{1}{2}\right)}{\rho^{2}} \tilde{\gamma}\right\}, \\
& \mathcal{M}[1,4]=0, \\
& \mathcal{M}[1,5]=-\frac{1}{\sqrt{6}}\left\{\frac{\partial}{\partial \rho} C_{1} \frac{\partial}{\partial z}-\frac{\partial}{\partial z} C_{2} \frac{\partial}{\partial \rho}+\frac{\left(F_{z}-\frac{1}{2}\right)}{\rho}\left[C_{1} \frac{\partial}{\partial z}-\frac{\partial}{\partial z} C_{2}\right]\right\}, \\
& \mathcal{M}[1,6]=-\sqrt{6}\left\{\frac{\partial}{\partial \rho} \tilde{\gamma} \frac{\partial}{\partial \rho}+\frac{\left(F_{z}+\frac{1}{2}\right)}{\rho} \frac{\partial}{\partial \rho} \tilde{\gamma}+\frac{\left(F_{z}-\frac{1}{2}\right)}{\rho} \tilde{\gamma} \frac{\partial}{\partial \rho}+\frac{\left(F_{z}-\frac{3}{2}\right)\left(F_{z}+\frac{1}{2}\right)}{\rho^{2}} \tilde{\gamma}\right\}, \\
& \mathcal{M}[2,1]=\frac{1}{\sqrt{3}}\left\{\frac{\partial}{\partial z} C_{1} \frac{\partial}{\partial \rho}-\frac{\partial}{\partial \rho} C_{2} \frac{\partial}{\partial z}+\frac{\left(F_{z}-\frac{3}{2}\right)}{\rho}\left[C_{2} \frac{\partial}{\partial z}-\frac{\partial}{\partial z} C_{1}\right]\right\}, \\
& \mathcal{M}[2,2]=\frac{\partial}{\partial \rho}\left(\gamma_{1}-\gamma_{2}\right) \frac{\partial}{\partial \rho}+\frac{\left(\gamma_{1}-\gamma_{2}\right)}{\rho} \frac{\partial}{\partial \rho}+\frac{\partial}{\partial z}\left(\gamma_{1}+2 \gamma_{2}\right) \frac{\partial}{\partial z}-\frac{\left(F_{z}-\frac{1}{2}\right)^{2}}{\rho^{2}}\left(\gamma_{1}-\gamma_{2}\right)+ \\
& \frac{\left(F_{z}-\frac{1}{2}\right)}{6 \rho}\left[\frac{\partial}{\partial \rho}\left(C_{1}+C_{2}\right)-\left(C_{1}+C_{2}\right) \frac{\partial}{\partial \rho}\right]-2\left(\gamma_{1}-\gamma_{2}\right)\left[\frac{\left(F_{z}-\frac{1}{6}\right) B}{2}+\frac{B^{2} \rho^{2}}{8}\right], \\
& \mathcal{M}[2,3]=\frac{1}{3}\left\{\frac{\partial}{\partial \rho}\left(C_{1}+C_{2}\right) \frac{\partial}{\partial z}-\frac{\partial}{\partial z}\left(C_{1}+C_{2}\right) \frac{\partial}{\partial \rho}+\frac{\left(F_{z}+\frac{1}{2}\right)}{\rho}\left[\left(C_{1}+C_{2}\right) \frac{\partial}{\partial z}-\frac{\partial}{\partial z}\left(C_{1}+C_{2}\right)\right]\right\}, \\
& \mathcal{M}[2,4]=\sqrt{3}\left\{\frac{\partial}{\partial \rho} \tilde{\gamma} \frac{\partial}{\partial \rho}+\frac{\left(F_{z}+\frac{3}{2}\right)}{\rho} \frac{\partial}{\partial \rho} \tilde{\gamma}+\frac{\left(F_{z}+\frac{1}{2}\right)}{\rho} \tilde{\gamma} \frac{\partial}{\partial \rho}+\frac{\left(F_{z}+\frac{3}{2}\right)\left(F_{z}-\frac{1}{2}\right)}{\rho^{2}} \tilde{\gamma}\right\}, \\
& \mathcal{M}[2,5]=\sqrt{2}\left\{\frac{\partial}{\partial \rho} \gamma_{2} \frac{\partial}{\partial \rho}-2 \frac{\partial}{\partial z} \gamma_{2} \frac{\partial}{\partial z}+\frac{\gamma_{2}}{\rho} \frac{\partial}{\partial \rho}-\frac{\left(F_{z}-\frac{1}{2}\right)^{2}}{\rho^{2}} \gamma_{2}+\right. \\
& \left.\frac{\left(F_{z}-\frac{1}{2}\right)}{6 \rho}\left[\frac{\partial}{\partial \rho}\left(C_{1}+C_{2}\right)-\left(C_{1}+C_{2}\right) \frac{\partial}{\partial \rho}\right]\right\}-2 \gamma_{2} \frac{B}{3}, \\
& \mathcal{M}[2,6]=-\frac{1}{3 \sqrt{2}}\left\{\frac{\partial}{\partial \rho}\left(C_{1}-2 C_{2}\right) \frac{\partial}{\partial z}+\frac{\partial}{\partial z}\left(2 C_{1}-C_{2}\right) \frac{\partial}{\partial \rho}+\frac{\left(F_{z}+\frac{1}{2}\right)}{\rho}\left[\left(C_{1}-2 C_{2}\right) \frac{\partial}{\partial z}+\frac{\partial}{\partial z}\left(2 C_{1}-C_{2}\right)\right]\right\}, \\
& \mathcal{M}[3,1]=-\sqrt{3}\left\{\frac{\partial}{\partial \rho} \tilde{\gamma} \frac{\partial}{\partial \rho}-\frac{\left(F_{z}-\frac{3}{2}\right)}{\rho} \frac{\partial}{\partial \rho} \tilde{\gamma}-\frac{\left(F_{z}-\frac{1}{2}\right)}{\rho} \tilde{\gamma} \frac{\partial}{\partial \rho}+\frac{\left(F_{z}-\frac{3}{2}\right)\left(F_{z}+\frac{1}{2}\right)}{\rho^{2}} \tilde{\gamma}\right\}, \\
& \mathcal{M}[3,2]=\frac{1}{3}\left\{\frac{\partial}{\partial z}\left(C_{1}+C_{2}\right) \frac{\partial}{\partial \rho}-\frac{\partial}{\partial \rho}\left(C_{1}+C_{2}\right) \frac{\partial}{\partial z}+\frac{\left(F_{z}-\frac{1}{2}\right)}{\rho}\left[\left(C_{1}+C_{2}\right) \frac{\partial}{\partial z}-\frac{\partial}{\partial z}\left(C_{1}+C_{2}\right)\right]\right\}, \\
& \mathcal{M}[3,3]=\frac{\partial}{\partial \rho}\left(\gamma_{1}-\gamma_{2}\right) \frac{\partial}{\partial \rho}+\frac{\left(\gamma_{1}-\gamma_{2}\right)}{\rho} \frac{\partial}{\partial \rho}+\frac{\partial}{\partial z}\left(\gamma_{1}+2 \gamma_{2}\right) \frac{\partial}{\partial z}-\frac{\left(F_{z}+\frac{1}{2}\right)^{2}}{\rho^{2}}\left(\gamma_{1}-\gamma_{2}\right)- \\
& \frac{\left(F_{z}+\frac{1}{2}\right)}{6 \rho}\left[\frac{\partial}{\partial \rho}\left(C_{1}+C_{2}\right)-\left(C_{1}+C_{2}\right) \frac{\partial}{\partial \rho}\right]-2\left(\gamma_{1}-\gamma_{2}\right)\left[\frac{\left(F_{z}+\frac{1}{6}\right) B}{2}+\frac{B^{2} \rho^{2}}{8}\right], \\
& \mathcal{M}[3,4]=\frac{1}{\sqrt{3}}\left\{\frac{\partial}{\partial z} C_{1} \frac{\partial}{\partial \rho}-\frac{\partial}{\partial \rho} C_{2} \frac{\partial}{\partial z}-\frac{\left(F_{z}+\frac{3}{2}\right)}{\rho}\left[C_{2} \frac{\partial}{\partial z}-\frac{\partial}{\partial z} C_{1}\right]\right\}, \\
& \mathcal{M}[3,5]=\frac{1}{3 \sqrt{2}}\left\{\frac{\partial}{\partial \rho}\left(C_{1}-2 C_{2}\right) \frac{\partial}{\partial z}+\frac{\partial}{\partial z}\left(2 C_{1}-C_{2}\right) \frac{\partial}{\partial \rho}-\frac{\left(F_{z}-\frac{1}{2}\right)}{\rho}\left[\left(C_{1}-2 C_{2}\right) \frac{\partial}{\partial z}+\frac{\partial}{\partial z}\left(2 C_{1}-C_{2}\right)\right]\right\},
\end{aligned}
$$




$$
\begin{aligned}
& \mathcal{M}[3,6]=\sqrt{2}\left\{\frac{\partial}{\partial \rho} \gamma_{2} \frac{\partial}{\partial \rho}-2 \frac{\partial}{\partial z} \gamma_{2} \frac{\partial}{\partial z}+\frac{\gamma_{2}}{\rho} \frac{\partial}{\partial \rho}-\frac{\left(F_{z}+\frac{1}{2}\right)^{2}}{\rho^{2}} \gamma_{2}-\right. \\
&\left.\frac{\left(F_{z}+\frac{1}{2}\right)}{6 \rho}\left[\frac{\partial}{\partial \rho}\left(C_{1}+C_{2}\right)-\left(C_{1}+C_{2}\right) \frac{\partial}{\partial \rho}\right]\right\}+2 \gamma_{2} \frac{B}{3},
\end{aligned}
$$

$\mathcal{M}[4,1]=0$,

$\mathcal{M}[4,2]=\sqrt{3}\left\{\frac{\partial}{\partial \rho} \tilde{\gamma} \frac{\partial}{\partial \rho}-\frac{\left(F_{z}-\frac{1}{2}\right)}{\rho} \frac{\partial}{\partial \rho} \tilde{\gamma}-\frac{\left(F_{z}+\frac{1}{2}\right)}{\rho} \tilde{\gamma} \frac{\partial}{\partial \rho}+\frac{\left(F_{z}+\frac{3}{2}\right)\left(F_{z}-\frac{1}{2}\right)}{\rho^{2}} \tilde{\gamma}\right\}$,

$\mathcal{M}[4,3]=\frac{1}{\sqrt{3}}\left\{\frac{\partial}{\partial \rho} C_{1} \frac{\partial}{\partial z}-\frac{\partial}{\partial z} C_{2} \frac{\partial}{\partial \rho}-\frac{\left(F_{z}+\frac{1}{2}\right)}{\rho}\left[C_{1} \frac{\partial}{\partial z}-\frac{\partial}{\partial z} C_{2}\right]\right\}$,

$\mathcal{M}[4,4]=\frac{\partial}{\partial \rho}\left(\gamma_{1}+\gamma_{2}\right) \frac{\partial}{\partial \rho}+\frac{\left(\gamma_{1}+\gamma_{2}\right)}{\rho} \frac{\partial}{\partial \rho}+\frac{\partial}{\partial z}\left(\gamma_{1}-2 \gamma_{2}\right) \frac{\partial}{\partial z}-\frac{\left(F_{z}+\frac{3}{2}\right)^{2}}{\rho^{2}}\left(\gamma_{1}+\gamma_{2}\right)-$

$\frac{\left(F_{z}+\frac{3}{2}\right)}{2 \rho}\left[\frac{\partial}{\partial \rho}\left(C_{1}+C_{2}\right)-\left(C_{1}+C_{2}\right) \frac{\partial}{\partial \rho}\right]-2\left(\gamma_{1}+\gamma_{2}\right)\left[\frac{\left(F_{z}+\frac{1}{2}\right) B}{2}+\frac{B^{2} \rho^{2}}{8}\right]$,

$\mathcal{M}[4,5]=\sqrt{6}\left\{\frac{\partial}{\partial \rho} \tilde{\gamma} \frac{\partial}{\partial \rho}-\frac{\left(F_{z}-\frac{1}{2}\right)}{\rho} \frac{\partial}{\partial \rho} \tilde{\gamma}-\frac{\left(F_{z}+\frac{1}{2}\right)}{\rho} \tilde{\gamma} \frac{\partial}{\partial \rho}+\frac{\left(F_{z}+\frac{3}{2}\right)\left(F_{z}-\frac{1}{2}\right)}{\rho^{2}} \tilde{\gamma}\right\}$,

$\mathcal{M}[4,6]=-\frac{1}{\sqrt{6}}\left\{\frac{\partial}{\partial \rho} C_{1} \frac{\partial}{\partial z}-\frac{\partial}{\partial z} C_{2} \frac{\partial}{\partial \rho}-\frac{\left(F_{z}+\frac{1}{2}\right)}{\rho}\left[C_{1} \frac{\partial}{\partial z}-\frac{\partial}{\partial z} C_{2}\right]\right\}$,

$\mathcal{M}[5,1]=-\frac{1}{\sqrt{6}}\left\{\frac{\partial}{\partial z} C_{1} \frac{\partial}{\partial \rho}-\frac{\partial}{\partial \rho} C_{2} \frac{\partial}{\partial z}+\frac{\left(F_{z}-\frac{3}{2}\right)}{\rho}\left[C_{2} \frac{\partial}{\partial z}-\frac{\partial}{\partial z} C_{1}\right]\right\}$,

$\mathcal{M}[5,2]=\sqrt{2}\left\{\frac{\partial}{\partial \rho} \gamma_{2} \frac{\partial}{\partial \rho}-2 \frac{\partial}{\partial z} \gamma_{2} \frac{\partial}{\partial z}+\frac{\gamma_{2}}{\rho} \frac{\partial}{\partial \rho}-\frac{\left(F_{z}-\frac{1}{2}\right)^{2}}{\rho^{2}} \gamma_{2}+\right.$

$$
\left.\frac{\left(F_{z}-\frac{1}{2}\right)}{6 \rho}\left[\frac{\partial}{\partial \rho}\left(C_{1}+C_{2}\right)-\left(C_{1}+C_{2}\right) \frac{\partial}{\partial \rho}\right]\right\}-2 \gamma_{2} \frac{B}{3},
$$

$\mathcal{M}[5,3]=\frac{1}{3 \sqrt{2}}\left\{\frac{\partial}{\partial \rho}\left(2 C_{1}-C_{2}\right) \frac{\partial}{\partial z}+\frac{\partial}{\partial z}\left(C_{1}-2 C_{2}\right) \frac{\partial}{\partial \rho}+\frac{\left(F_{z}+\frac{1}{2}\right)}{\rho}\left[\left(2 C_{1}-C_{2}\right) \frac{\partial}{\partial z}+\frac{\partial}{\partial z}\left(C_{1}-2 C_{2}\right)\right]\right\}$,

$\mathcal{M}[5,4]=\sqrt{6}\left\{\frac{\partial}{\partial \rho} \tilde{\gamma} \frac{\partial}{\partial \rho}+\frac{\left(F_{z}+\frac{3}{2}\right)}{\rho} \frac{\partial}{\partial \rho} \tilde{\gamma}+\frac{\left(F_{z}+\frac{1}{2}\right)}{\rho} \tilde{\gamma} \frac{\partial}{\partial \rho}+\frac{\left(F_{z}+\frac{3}{2}\right)\left(F_{z}-\frac{1}{2}\right)}{\rho^{2}} \tilde{\gamma}\right\}$,

$\mathcal{M}[5,5]=\frac{\partial}{\partial \rho} \gamma_{1} \frac{\partial}{\partial \rho}+\frac{\partial}{\partial z} \gamma_{1} \frac{\partial}{\partial z}+\frac{\gamma_{1}}{\rho} \frac{\partial}{\partial \rho}-\frac{\left(F_{z}-\frac{1}{2}\right)^{2}}{\rho^{2}} \gamma_{1}+$

$$
\frac{\left(F_{z}-\frac{1}{2}\right)}{3 \rho}\left[\frac{\partial}{\partial \rho}\left(C_{1}+C_{2}\right)-\left(C_{1}+C_{2}\right) \frac{\partial}{\partial \rho}\right]-2 \gamma_{1}\left[\frac{\left(F_{z}+\frac{1}{6}\right) B}{2}+\frac{B^{2} \rho^{2}}{8}\right]-2 \Delta_{o}(\rho, z),
$$

$\mathcal{M}[5,6]=-\frac{1}{3}\left\{\frac{\partial}{\partial \rho}\left(C_{1}+C_{2}\right) \frac{\partial}{\partial z}-\frac{\partial}{\partial z}\left(C_{1}+C_{2}\right) \frac{\partial}{\partial \rho}+\frac{\left(F_{z}+\frac{1}{2}\right)}{\rho}\left[\left(C_{1}+C_{2}\right) \frac{\partial}{\partial z}-\frac{\partial}{\partial z}\left(C_{1}+C_{2}\right)\right]\right\}$,

$\mathcal{M}[6,1]=-\sqrt{6}\left\{\frac{\partial}{\partial \rho} \tilde{\gamma} \frac{\partial}{\partial \rho}-\frac{\left(F_{z}-\frac{3}{2}\right)}{\rho} \frac{\partial}{\partial \rho} \tilde{\gamma}-\frac{\left(F_{z}-\frac{1}{2}\right)}{\rho} \tilde{\gamma} \frac{\partial}{\partial \rho}+\frac{\left(F_{z}-\frac{3}{2}\right)\left(F_{z}+\frac{1}{2}\right)}{\rho^{2}} \tilde{\gamma}\right\}$,

$\mathcal{M}[6,2]=-\frac{1}{3 \sqrt{2}}\left\{\frac{\partial}{\partial \rho}\left(2 C_{1}-C_{2}\right) \frac{\partial}{\partial z}+\frac{\partial}{\partial z}\left(C_{1}-2 C_{2}\right) \frac{\partial}{\partial \rho}-\frac{\left(F_{z}-\frac{1}{2}\right)}{\rho}\left[\left(2 C_{1}-C_{2}\right) \frac{\partial}{\partial z}+\frac{\partial}{\partial z}\left(C_{1}-2 C_{2}\right)\right]\right\}$,

$\mathcal{M}[6,3]=\sqrt{2}\left\{\frac{\partial}{\partial \rho} \gamma_{2} \frac{\partial}{\partial \rho}-2 \frac{\partial}{\partial z} \gamma_{2} \frac{\partial}{\partial z}+\frac{\gamma_{2}}{\rho} \frac{\partial}{\partial \rho}-\frac{\left(F_{z}+\frac{1}{2}\right)^{2}}{\rho^{2}} \gamma_{2}-\right.$

$$
\left.\frac{\left(F_{z}+\frac{1}{2}\right)}{6 \rho}\left[\frac{\partial}{\partial \rho}\left(C_{1}+C_{2}\right)-\left(C_{1}+C_{2}\right) \frac{\partial}{\partial \rho}\right]\right\}+2 \gamma_{2} \frac{B}{3},
$$

$\mathcal{M}[6,4]=-\frac{1}{\sqrt{6}}\left\{\frac{\partial}{\partial z} C_{1} \frac{\partial}{\partial \rho}-\frac{\partial}{\partial \rho} C_{2} \frac{\partial}{\partial z}-\frac{\left(F_{z}+\frac{3}{2}\right)}{\rho}\left[C_{2} \frac{\partial}{\partial z}-\frac{\partial}{\partial z} C_{1}\right]\right\}$,

$\mathcal{M}[6,5]=-\frac{1}{3}\left\{\frac{\partial}{\partial z}\left(C_{1}+C_{2}\right) \frac{\partial}{\partial \rho}-\frac{\partial}{\partial \rho}\left(C_{1}+C_{2}\right) \frac{\partial}{\partial z}+\frac{\left(F_{z}-\frac{1}{2}\right)}{\rho}\left[\left(C_{1}+C_{2}\right) \frac{\partial}{\partial z}-\frac{\partial}{\partial z}\left(C_{1}+C_{2}\right)\right]\right\}$,

$\mathcal{M}[6,6]=\frac{\partial}{\partial \rho} \gamma_{1} \frac{\partial}{\partial \rho}+\frac{\partial}{\partial z} \gamma_{1} \frac{\partial}{\partial z}+\frac{\gamma_{1}}{\rho} \frac{\partial}{\partial \rho}-\frac{\left(F_{z}+\frac{1}{2}\right)^{2}}{\rho^{2}} \gamma_{1}-$

$$
\frac{\left(F_{z}+\frac{1}{2}\right)}{3 \rho}\left[\frac{\partial}{\partial \rho}\left(C_{1}+C_{2}\right)-\left(C_{1}+C_{2}\right) \frac{\partial}{\partial \rho}\right]-2 \gamma_{1}\left[\frac{\left(F_{z}-\frac{1}{6}\right) B}{2}+\frac{B^{2} \rho^{2}}{8}\right]-2 \Delta_{o}(\rho, z) .
$$


Here $\gamma_{i}$ are the position-dependent Luttinger parameters, $\tilde{\gamma}=\left(\gamma_{2}+\gamma_{3}\right) / 2, C_{1}=$ $1+\gamma_{1}-2 \gamma_{2}-6 \gamma_{3}$ and $C_{2}=1+\gamma_{1}-2 \gamma_{2}, F_{z}$ is the total angular momentum $z$-projection and $\Delta_{o}(\rho, z)$ the spin-orbit splitting.

\section{References}

[1] Lefebvre P, Gayral B, 2008 Comptes Rendus Physique 9816.

[2] Weng M Q, Wang Y Y, and Wu M W, Phys. Rev. B 200979155309.

[3] Martinez-Guerrero E, Adelmann C, Chabuel F, Simon J, Pelekanos N T, Mula G, Daudin B, Feuillet G. and Mariette H, 2000 Appl. Phys. Lett. 77809.

[4] Martinez-Guerrero E, Chabuel F, Daudin B, Rouviere J L and Mariette H, 2002 Appl. Phys. Lett. 815117.

[5] Fonoberov V A and Balandin A A, 2003 J. Appl. Phys. 947178.

[6] Simon J, Pelekanos N T, Adelmann C, Martinez-Guerrero E, André R, Daudin B, Dang L S and Mariette H, 2003 Phys. Rev. B 68035312.

[7] Shojaei S, Troiani F, Asgari A, Kalafi M, and Goldoni G, 2008 The European Physical Journal B 65505.

[8] Nagahara S, Arita M, and Arakawa Y, 2006 Appl. Phys. Lett. 88083101.

[9] Lagarde D, Balocchi A, Carrere H, Renucci P, Amand T, Marie X, Founta S and Mariette H, 2008 Phys. Rev. B 77 041304(R).

[10] Brimont C, Gallart M, Cregut O, Hoenerlage B, Gilliot P, Lagarde D, Balocchi A, Amand T, Marie X, Founta S, and Mariette H, 2009 J. Appl. Phys. 106053514.

[11] Buss J H, Rudolph J, Schupp T, As D J, Lischka K and Haegele D, 2010 Appl. Phys. Lett. 97 062101.

[12] Xia C, Zeng Z and Wei S, 2011 Journal of luminescence 131623.

[13] Tong H and Wu M W, 2011 Phys. Rev. B 83235323.

[14] Chuang S L and Chang C S, 1996 Appl. Phys. Lett. 681657.

[15] Liao Y H, Climente J I and Cheng S J, 2011 Phys. Rev. B 83165317.

[16] Tsitsishvili E and Kalt H, 2010 Phys. Rev. B 82195315.

[17] Vurgaftman I, Meyer J L and Ram-Mohan L R, 2001 J. Appl. Phys. 895815.

[18] Rodrigues S C P, Scolfaro L M R, Leite J R and Sipahi G M, 2000 Appl. Phys. Lett. 761015.

[19] Marquardt O, Mourad D, Schulz S, Hickel T, Czycholl G and Neugebauer J, 2008 Phys. Rev. B 78235302.

[20] Luttinger J M, and Kohn W, 1955 Phys. Rev. 97, 869; Luttinger J M, 1956 ibid. 102, 1030.

[21] Burt M G, 1992 J. Phys.: Condens. Matter 4, 6651; 1999 ibid. 11, R53.

[22] Foreman B A, 1993 Phys. Rev. B 48, 4964.

[23] Voon L and Willatzen M, The k.p Method: Electronic Properties of Semiconductors (Springer Verlag, 2009).

[24] Please note that Table 12.4 in ref. [23], where the information is included, contains a few typos. Thus, the Hamiltonian element $(5,4)$ must be $+\sqrt{2} R$ instead of $-\sqrt{2} R$, the Hamiltonian element $(6,3)$ should be $-\sqrt{2} Q^{*}$ instead of $-\sqrt{2} Q$. Finally, in the definitions of $Q$, the third term in the sum reading $+2 k_{z}(L-M) k_{z}$ must be $-2 k_{z}(L-M) k_{z}$.

[25] Planelles J, and Jaskólski W, 2003 J. of Phys.: Condens. Matter 15, L67.

[26] Mlinar V, Tadic M, Partoens B and Peeters F M, 2005 Phys. Rev. B 71, 205305.

[27] Climente J I, Planelles J, and Jaskólski W, 2003 Phys. Rev. B 68075307.

[28] Planelles J, Climente J I, Rajadell F, Doty M F, Bracker A S and Gammon D, 2010 Phys. Rev. B 82155307.

[29] Sercel P C and Vahala K J, 1990 Phys. Rev. B 42, 3690.

[30] Mietze C, Landmann M, Rauls E, Machhadani H, Sakr S, Tchernycheva M, Julien F H, Schmidt W G, Lischka K and As D J, 2011 Phys. Rev. B 83195301. 
[31] The analysis is analogous for the Kramers-degenerate $F z=-3 / 2$ state.

[32] Hu J, Li L, Yang W, Manna L, Wang L and Alivisatos A, 2001 Science 2922060.

[33] Planelles J, Rajadell F and Climente J I, 2010 J. Phys. Chem. C 1148337.

[34] Baskoutas S and Bester G, 2011 J. Phys. Chem. C 115 15862; 2010 ibid 11494301.

[35] Climente J I, Planelles J, Pi M and Malet F, 2005 Phys. Rev. B 72233305.

[36] Koudinov A V, Akimov I A, Kusrayev Y G and Henneberger F, 2004 Phys. Rev. B 70241305.

[37] Bardoux R, Guillet T, Gil B, Lefebvre P, Bretagnon T, Taliercio T, Rousset S, and Semond F, 2008 Phys. Rev. B $\mathbf{7 7} 235315$.

[38] Belhadj T, Amand T, Kunold A, Simon C M, Kuroda T, Abbarchi M, Mano T, Sakoda K, Kunz S, Marie X, and Urbaszek B, 2010 Appl. Phys. Lett. 97051111.

[39] Doty M F, Climente J I, Greilich A, Yakes M, Bracker A S and Gammon D, 2010 Phys. Rev. B 81035308.

[40] Keizer J G, Bocquel J, Koenraad P M, Mano T, Noda T, and Sakoda K, 2010 Appl. Phys. Lett. 96062101. 